Supporting Information

\title{
Water Layer at Hydrophobic Surface: Electrically Dead but Dynamically Alive?
}

\author{
Sayantan Mondal and Biman Bagchi* \\ Solid State and Structural Chemistry Unit \\ Indian Institute of Science, Bengaluru \\ Karnataka 560 012, India \\ *Email: bbagchi@iisc.ac.in
}

\section{System and simulation details}

We perform atomistic molecular dynamics simulations of three different shapes of nanoconfined water systems, namely, (a) nano-spheres, (b) nano-cylinders, and (c) nano-slabs. The force-field parameters (Table S1) and simulation details are provided below.

Table S1. The force-field parameters for surface atoms and SPC/E water. The water molecules are nonpolarisable and possess a permanent dipole moment of 2.35 debye whereas, the hydrophobic wall atoms are modeled as non-polarisable Lennard-Jones particles.

\begin{tabular}{|c|c|c|c|c|c|}
\hline & $\sigma_{L J}(\mathrm{~nm})$ & $\varepsilon_{L J}\left(\mathrm{kCal} \mathrm{mol}^{-1}\right)$ & $q / e$ & bond length $(\mathrm{nm})$ & Angle \\
\hline Water & 0.316 & 0.155 & $\begin{array}{l}\mathrm{q}_{\mathrm{o}}=-0.8476, \\
\mathrm{q}_{\mathrm{H}}=+0.4238\end{array}$ & $d_{O-H}=0.10$ & $\theta_{H \hat{O} H}=109.47^{\circ}$ \\
$\begin{array}{c}\text { Surface } \\
\text { atoms }\end{array}$ & 0.34 & 0.09 & uncharged & $d_{C-C}=0.142$ & $\theta_{C \hat{C} C}=120^{\circ}$ \\
\hline
\end{tabular}

Throughout these simulations, the graphene wall atoms are kept frozen. We have used leap-frog integrator with 2 fs time step to propagate the systems in NVT ensembles $(\mathrm{T}=300$ $\mathrm{K})$. The temperature of all these systems is kept fixed with Nose-Hoover thermostat ( $\tau=0.21 \mathrm{ps}^{-1}$ ). We use particle mesh Ewald to obtain long-range electrostatics with an FFT grid spacing of $0.16 \mathrm{~nm}$ along the periodic directions. The trajectories are $10 \mathrm{~ns}$ long with $4 \mathrm{fs}$ data dumping rate. All simulations are performed using GROMACS. ${ }^{1}$

We have simulated spherical nano-cavity of radius $(R)=1.5 \mathrm{~nm}$. We have filled up the cavity by following the energy criterion with a certain degree of allowed overlap (tolerance $\sim 0.2 \mathrm{~nm}$ ). The simulations are performed without any periodic boundaries. 
We have modeled a single-walled carbon nano tube (CNT)-water system of fixed length $(\mathrm{L}=5 \mathrm{~nm})$ and diameter $1.6 \mathrm{~nm}\left(\mathrm{~N}_{\mathrm{wat}}=198\right)$. We have employed periodic boundaries in the Zdirection only, which makes it an infinitely long CNT along Z. We have inserted the average number of water molecules $\left(N_{\text {wat }}\right)$ obtained from another set of $10 \mathrm{~ns}$ long equilibrium MD trajectories where the cylinder has been immersed inside a tetragonal box filled with SPC/E water molecules so that a water bath is present.

We have simulated a nanoslab systems filled with SPC/E water with $d=3.0 \mathrm{~nm}$ $\left(\mathrm{N}_{\mathrm{wat}}=2,301\right)$. Here $d$ is the distance of separation between two square slabs (perpendicular to the Z-axis). We have chosen the dimensions of the slabs as $5 \mathrm{~nm} \times 5 \mathrm{~nm}$. We find out the optimum number of water molecules inside the confinement from a separate set of $10 \mathrm{~ns}$ long NVT simulations where an external water bath is attached. We have imposed periodic boundary conditions (PBC) along the $\mathrm{X}$ and $\mathrm{Y}$ directions (parallel to the plane of the slabs) of the confined water systems.

\section{Diffusivity and free energy profiles}

We provide the shell-wise average residence times of water molecules in three nanoconfined water systems in the main text (Table 1). We find that the interfacial water layers (or the electrically dead layers) possess higher residence time compared to the successive water layers. However, the water molecules that reside at the core of the confining geometry also exhibit higher residence times. Here, we connect the observed residence times with diffusivity and free energy profiles. The position dependent average residence time (or the mean first passage times, $[\tau(\mathrm{r})])$, the position dependent diffusion coefficient $[\mathrm{D}(\mathrm{r})]$, and the free energy $[F(r)]$ are related by the following elegant expression. ${ }^{2}$

$$
\tau(r)=\int_{x}^{b} d y \frac{1}{D(y)} e^{\beta F(y)} \int_{a}^{y} d x e^{-\beta F(x)}
$$

Where an absorptive barrier is located at $\mathrm{r}=\mathrm{b}$, a reflective barrier is located at $\mathrm{r}=\mathrm{a}$, and $\beta=\left(k_{B} T\right)^{-1}$. We note that Eq. (1.1) is exact in one-dimensions and only approximately valid in higher dimensions.

However, it is immensely challenging to calculate zone-wise diffusion coefficients because of the short residence times and exchange among domains. The complexity increases even more as there is ruggedness in the energy landscape at the molecular length scale 
(Figure S1). We have also tried to obtain the diffusion coefficients conventionally from velocity autocorrelations. However, again due to the constraint of the short residence times we have a limited scope for averaging. As a result, the values become unreliable. Nevertheless, the difference in the initial decay between the parallel and perpendicular components of the interfacial layer is distinct. But it becomes noisy in the long time. Thus, it makes sense to obtain the time-dissected mean square displacements. These show interesting variations which seem to agree with free energy profiles.

We define a quantity $\Delta$ as a measure of the anisotropic diffusion. $\Delta$ denotes the average distance travelled by the water molecules in a particular grid within a time interval $\delta \tau$. Here, we have chosen $\delta \tau=5$ ps. Because of the short and finite residence times of water molecules, one cannot extract reliable numbers for smaller grids. Hence, we need to reduce the number of layers (that is, coarse-graining) to obtain the position-dependent $\Delta$.

In the non-spherical systems, namely, slab and nanotube, the diffusion becomes anisotropic. For the slab geometry, one can obtain $\Delta$ for water in the XY plane (that is, $\Delta_{\|}$) and along the $\mathrm{Z}$ direction (that is, $\Delta_{\perp}$ ). The free energy is constant along the $\mathrm{XY}$ plane for a fixed value of Z. Similarly for the cylindrical geometry one can obtain the axial (along the Zdirection, $\left.\Delta_{\mathrm{ax}}\right)$ and radial $\left(\Delta_{\mathrm{r}}\right)$ components. Here the free energy becomes constant along the

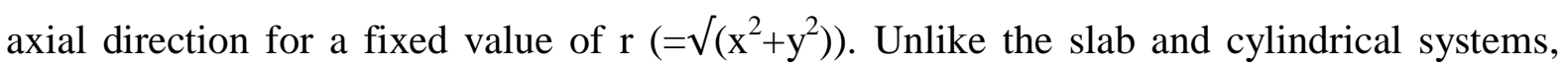
diffusion is isotropic in the spherical system. Therefore, in order to obtain the anisotropic $\Delta$, we coarse grain the systems with $5 \AA$ thick grids. We show the results in Table S2.

Table S2. Shell-wise anisotropic $\Delta$ for water molecules confined inside slab, cylinder, and spherical nanoenclosures. The values of $\Delta$ along the non-periodic (confined) directions, namely, $\Delta_{\perp}$ and $\Delta_{\mathrm{r}}$ are lower than that along the periodic (open) directions, namely, $\Delta_{\|}$and $\Delta_{\mathrm{ax}}$. The interfacial water molecules exhibit enhanced diffusion along the open directions compared to the subsequent water layers.

\begin{tabular}{|c|c|c|c|c|}
\hline System & $\Delta(\mathbf{n m})$ & $\begin{array}{c}\text { Shell-1 } \\
(\text { EDL })\end{array}$ & $\begin{array}{c}\text { Shell-2 } \\
\text { (Intermediate layers) }\end{array}$ & $\begin{array}{c}\text { Shell-3 } \\
\text { (Core layer) }\end{array}$ \\
\hline \multirow{2}{*}{ Slab } & $\Delta_{\|}$ & $0.291 \pm 0.012$ & $0.275 \pm 0.011$ & $0.255 \pm 0.010$ \\
\cline { 2 - 5 } & $\Delta_{\perp}$ & $0.153 \pm 0.012$ & $0.225 \pm 0.015$ & $0.230 \pm 0.016$ \\
\hline \multirow{2}{*}{ Cylinder } & $\Delta_{\mathrm{ax}}$ & $0.246 \pm 0.018$ & $0.255 \pm 0.020$ & $0.266 \pm 0.022$ \\
\cline { 2 - 5 } & $\Delta_{\mathrm{r}}$ & $0.268 \pm 0.011$ & $0.291 \pm 0.012$ & $0.284 \pm 0.014$ \\
\hline Sphere & $\Delta_{\mathrm{r}}$ & $0.267 \pm 0.008$ & $0.302 \pm 0.012$ & $0.310 \pm 0.015$ \\
\hline BULK & $\Delta$ & & $0.274 \pm 0.01$ & \\
\hline
\end{tabular}


We find that the diffusion is anisotropic for slab and cylindrical geometries, as discussed above. In the interfacial layer (EDL), the parallel (and the axial) component of $\Delta$ is larger than the successive water layers. At the same time, the perpendicular (and radial) components are substantially attenuated compared to the parallel (and axial) ones. We note that these values may not fully correlate with the residence times as those were also calculated by coarse graining. Below we connect these results with the free-energy profiles.

The free energy profiles $[\mathrm{F}(\mathrm{r})$ or $\mathrm{F}(\mathrm{z})]$ distinctly modulates the diffusivity profiles and water dynamics at the interface. We calculate the potential of mean force along the nonperiodic dimensions by using the following relations [Eqs. (1.2), (1.3), and (1.4)].

Slab geometry

$$
F(z)=-k_{B} T \ln \rho(z)
$$

Cylindrical geometry

$$
F(r)=-k_{B} T \ln \rho(r) ; r=\sqrt{\left(x^{2}+y^{2}\right)}
$$

Spherical geometry

$$
F(r)=-k_{B} T \ln \rho(r) ; r=\sqrt{\left(x^{2}+y^{2}+z^{2}\right)}
$$

Here, $\rho(\mathrm{r})$ or $\rho(\mathrm{z})$ are the normalized density profiles (or wall-water radial distribution functions) along the non-periodic directions. We show the results in Figure S1. It is clear from the results that the interfacial water molecules are trapped with substantially large freeenergy barriers. This partly restricts the translational motions of EDL water molecules along the perpendicular/radial directions. However, the free-energy is constant along the parallel (or the axial) direction and water molecules can exhibit their natural motion in the quasi twodimensional sub-systems distinctly separated by the free-energy barriers. It is noteworthy to mention that, in an earlier study, Netz and co-workers obtained the diffusivity and freeenergy profiles under parallel slab confinement with three different wall types. ${ }^{3}$ Our results corroborate well with the results of Netz et al. 

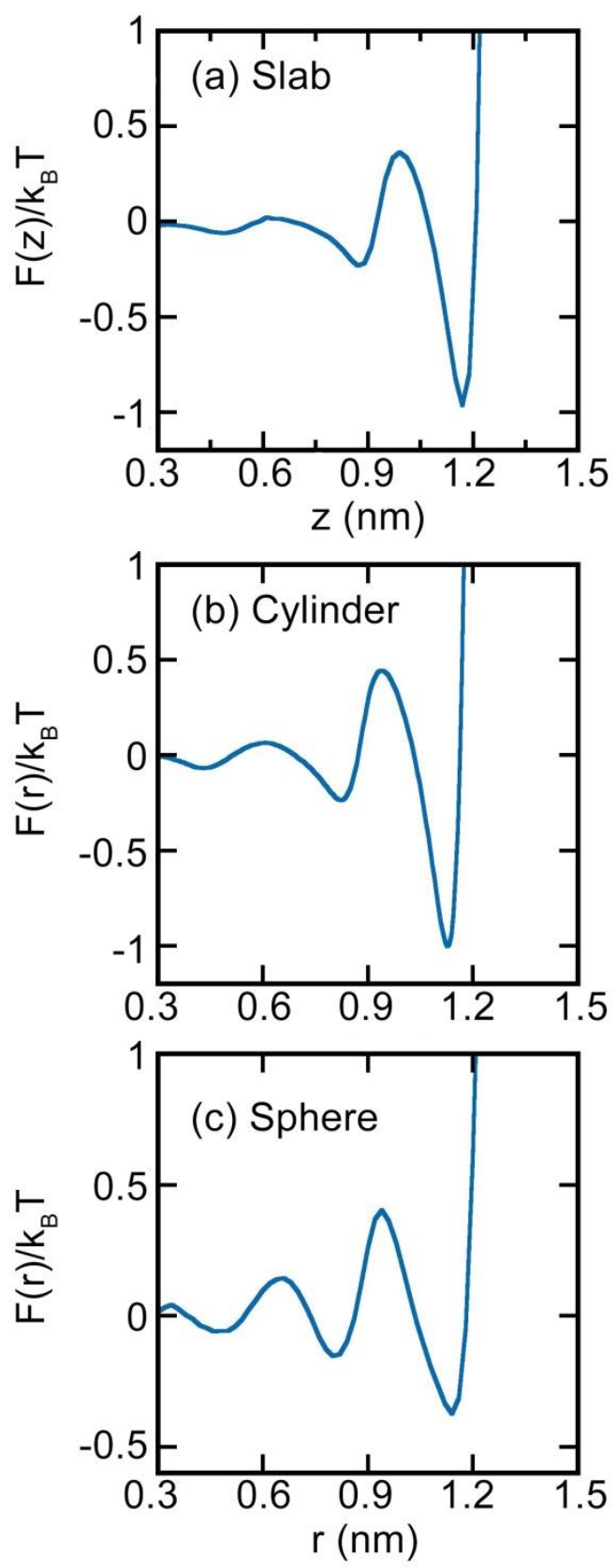

Figure S1. Potential of mean force (PMF) along the non-periodic directions for (a) slab, (b) cylindrical, and (c) spherical geometries. The interfacial layers are distinctly separated by a large free energy barrier. The height of the barrier influences the residence time of the water molecules that reside in the electrically dead layer. Here, $r$ and $z$ are measured from the centre of the confinement. 


\section{References}

1. Abraham, M. J.; Murtola, T.; Schulz, R.; Páll, S.; Smith, J. C.; Hess, B.; Lindahl, E.

GROMACS: High performance molecular simulations through multi-level parallelism from laptops to supercomputers, SoftwareX 1, 19-25 (2015).

2. Zwanzig, R. Nonequilibrium statistical mechanics. Oxford University Press: New York, USA. 2001.

3. Sedlmeier, F.; von Hansen, Y.; Mengyu, L.; Horinek, D.; Netz, R. R. Water dynamics at interfaces and solutes: disentangling free energy and diffusivity contributions, J. Stat. Phys. 145 (2), 240-252 (2011). 